\title{
Ceramic veneers for smile optimization: a multidisciplinary approach
}

\begin{abstract}
After orthodontic treatment, smile optimization seems to be challenging mainly for patients who are highly demanding and seek perfect esthetics with minimally invasive approaches. Lithium disilicate have been proved to be very efficient to attain satisfactory esthetics with predictable and lasting outcomes. This article describes a detailed clinical protocol of successful lithium disilicate veneers after an orthodontic treatment to establish appropriate tooth position and a mucogingival surgery to correct gingival margins, thus improving the gummy smile.
\end{abstract}

Keywords: esthetics, veneers, lithium disilicate
Volume 8 Issue | - 2018

\author{
I Azouzi, I Kalghoum, D Hadyaoui, B \\ Harzallah, M Cherif \\ Department of Fixed Prosthodontics, Faculty of dental medicine, \\ Tunisia \\ Correspondence: I Azouzi Department of Fixed \\ Prosthodontics, Research Laboratory of Occlusodontics and \\ Ceramic Prostheses LRI6ESI5, Faculty of Dental Medicine, \\ University of Monastir,Tunisia, \\ Email dalendaresearch@gmail.com
}

Received: December 29, 2017 | Published: January 09, 2018

\section{Introduction}

People have always sought an attractive smile in harmony with an esthetic appearance. This trend is steadily growing as it influences one's self esteem and psychological well-being. ${ }^{1,2}$ Faced with highly esthetic demanding patients, the practitioner should guarantee esthetic outcomes involving conservative procedures. This is undoubtedly challenging and often requiring a perfect multidisciplinary approach. ${ }^{3}$

Advances in physical and optical properties of ceramics and adhesive cementation techniques have definitely enabled to comply with three vital parameters: esthetic, function and bio economic principles. Thanks to its biocompatibility, biomimicry, longevity as well as its great resistance especially when reinforced with lithium disilicate, ceramic has been used. ${ }^{4}$

The outcomes have proved to be more predictable. Pneumas et al., 5 reported a survival rate ranging from $92 \%$ at 5 years to $64 \%$ at $10 y e a r s$. Beier et al., ${ }^{6}$ through their 20 -year retrospective study, estimated the survival rate of $90 \%$ at 12 to 13 years.

Lithium Disilicate veneers offers satisfactory esthetics with predictable and lasting outcomes as long as they are conducted following a meticulous treatment plan along with a strictly-applied protocol during the clinical and laboratory stages. ${ }^{3,7}$

What has really led to the fabrication of efficient and predictable ceramic restorations is the use of $\mathrm{CAD} / \mathrm{CAM}$ technologies during the processing. ${ }^{3}$

The article presents a well-illustrated protocol for the realization of ceramic veneers. Thus, improving the esthetic appearance with a multidisciplinary approach through a clinical report which involves anorthodontic treatment, a periodontal surgery and minimum thickness ceramic veneers.

\section{Case presentation}

A 30-year-old male patient who had undergone past orthodontic treatment was referred to the prosthodontics department with a chief complaint of unaesthetic smile. His medical history is noncontributory.

\section{Clinical examination}

A clinical and radiographic examination was carried out. Intraorally, tooth size discrepancies, improper clinical crown lengths and asymmetrical zenith lines were observed. In the maxillary arch, central incisors were predominant, lateral incisors and canine were remarkably smaller.

No dental decay was detected. Neither was bone/root resorption. From a periodontal perspective, the patient has quite satisfactory gingival health. He has a stable posterior occlusion after the orthodontic treatment (Figure 1). Whenever esthetic procedures are considered, emphasis must be put on gingival health. Thus, brushing technique had to be optimized. Periodontal treatment, including scaling and root planning should be first realized.

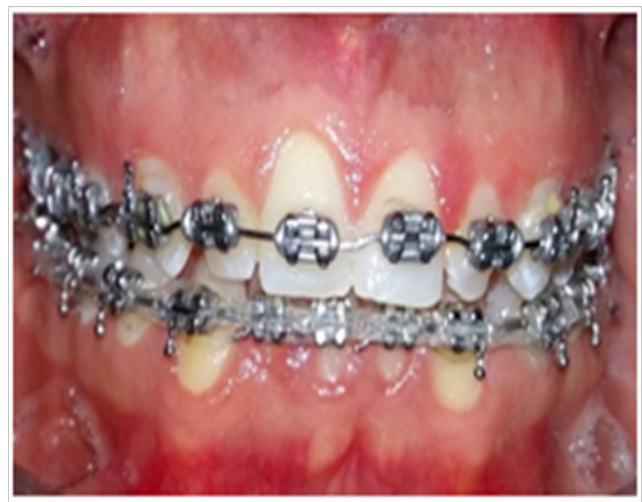

Figure I Preoperative view: final step of the orthodontic treatment.

In order to attain an optimal treatment plan, a photographic documentation and video clips were collected. Impressions for study templates were taken.

A meticulous analysis based on fundamental objective and subjective esthetic criteria involving the height-width ratio of the anterior teeth and the smile of the patient was performed. It revealed inadequate relative dimension of the anterior teeth as well as an imbalance of the dento-labial, dental and gingival complex. 


\section{The digital smile design:}

The digital smile design was performed to previsualize the esthetic outcome. The aim is to improve the communication with the patient, keep him involved throughout the planning of the smile and explain the advantages of the suggested treatment. There by motivating the patient to start the treatment.

\section{Mucogingival surgery}

The patient underwent gingivectomy to re-establish the correct position of the gingival collars mainly the zeniths as well as the appropriate clinical length/width ratio of the six anterior teeth (Figure 2).

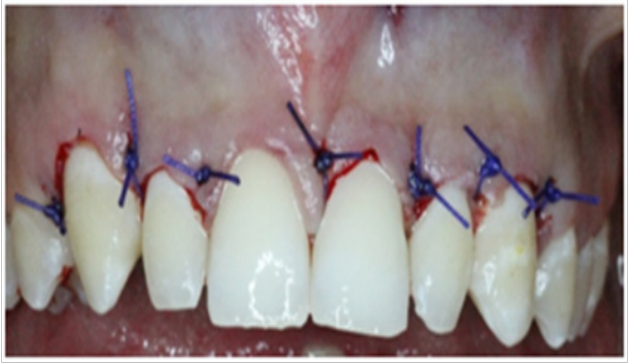

Figure 2 the gingivectomy from tooth \#13 to tooth \#23.

Two months later, periodontal healing was thoroughly achieved, gingival margins were stabilized and gum exposure during smile improved (Figure 3).

\section{The mock up}

The esthetic outcome is clinically previsualized through a direct mock up. The purpose is also to "deprogram" the patient from the previous situation. It remained in situ until the next appointment to ascertain esthetic, phonetics and function. In fact, the length and shape of the future restorations, the absence of interferences, the correct pronunciation as well as the patient comfort were assessed.

Few corrections were required during this step to live up to the patient's esthetic expectations.

\section{Prosthetic decision}

Owing to its conservative aspect and excellent esthetic result, the decision to perform lithium disilicate veneers from tooth \#13 to tooth \#23 was taken with the patient's consent. Although the microdontia of the mandibular anterior teeth, the patient showed disinterest in their restoration as he did not think it presented an esthetic problem.

\section{Shade selection}

Prior to tooth preparation, shade was selected using VITA master shade guide considering neighboring and opposite teeth

\section{Material selection}

We opted for lithium disilicate ceramic for its optical and mechanical properties and its high biocompatibility which would offer us promising esthetic results.

\section{Teeth preparation}

An extremely conservative preparation limited to enamel and driven by the mock up, was conducted without local anesthesia to generate enough space for ceramic to guarantee an adequate emergence profile and avoid over contour. Thus, periodontal health might be maintained.
Indeed, in the buccal face, $0.3 \mathrm{~mm}$ in the gingival third, $0.5 \mathrm{~mm}$ in the middle third and $0.8 \mathrm{~mm}$ in the incisal third were provided by means of calibrated depth cuts through the mock up. Then, vertical preparation between the depth grooves was realized to remove the dental substance by a round-end tapered diamond bur.

The finishing line was of curved chamfer design, para-gingivally localized and inter-proximally extended to cover visually accessible tooth areas. Contact points were preserved. Incisal preparation was performed over the incisal edge from buccal to palatal with $1.5 \mathrm{~mm}$ of incisal reduction. Due care was taken to round sharp angles especially incisaledges where the stress may be greatest, and to remove undercuts (Figure 4).

Two months later, periodontal healing was thoroughly achieved, gingival margins were stabilized and gum exposure during smile improved (Figure 3).

Eventually, preparations were meticulously finished and polished by means of yellow- tapered- diamond bur and a rubber cup.

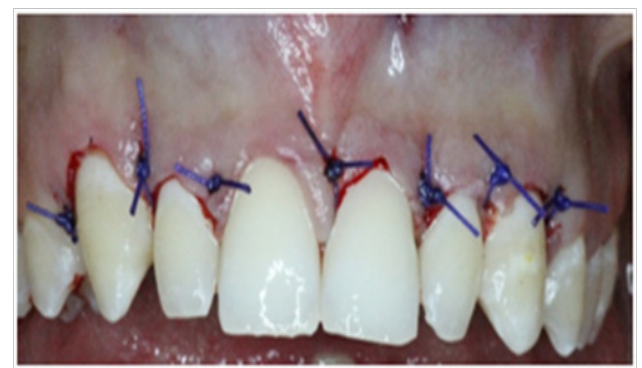

Figure 3 Intra oral images showing tooth size discrepancies.

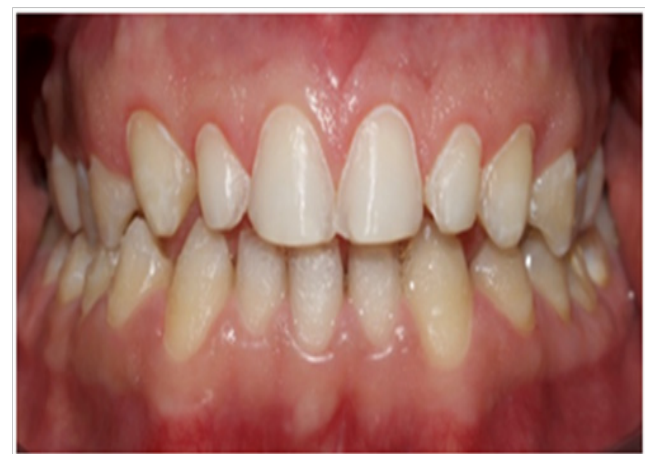

Figure 4 Extremely conservative tooth preparation within enamel from tooth \#| 3 to tooth \#23.

\section{Temporization}

Although preparation was within enamel, provisionalization was deemed necessary as it improved the patient self-confidence. It also gave him a preview of the final result.

\section{Impression procedure}

A one-phase impression, after retraction cords placement, was taken with putty and light body poly vinyl siloxane material. This ensured an accurate and complete reproduction of the entire margins and helped the dental technician obtain the correct emergence profile for the restorations.

Once the working impression is validated, the dental laboratory processed veneers by $\mathrm{CAD} / \mathrm{CAM}$ system (Figure 5). The characterization of the buccal surfaces was carried out using the cut- 
back technique requiring a surface ceramique scraping in favor of dental ceramic stratification.

Two months later, periodontal healing was thoroughly achieved, gingival margins were stabilized and gum exposure during smile improved (Figure 3).

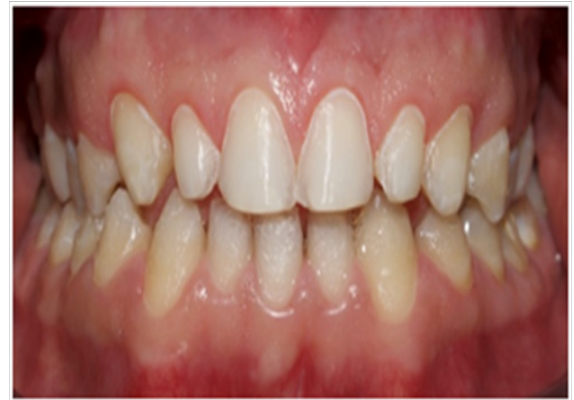

Figure 5 Digital model with virtual veneers.

\section{Try in protocol}

Removal of the provisional restorations was straightforward Tooth surfaces were thoroughly cleaned. Each veneer was tried on the prepared tooth.

All these parameters were checked: precise marginal fit, correct length and mesio-distal dimension, adequate contour in harmony with the surrounding tissues, color mimicry to natural adjacent teeth and proper esthetics improving the patient's smile.

The adhesive cementation was programmed in the following appointment.

\section{A step by step bonding procedure:}

The rubber dam, key to success to bonding procedure, was carefully positioned between the first two premolars to achieve adequate isolation (Figure 6). Extreme care was taken during the bonding protocol. The following sequenced steps were meticulously considered:

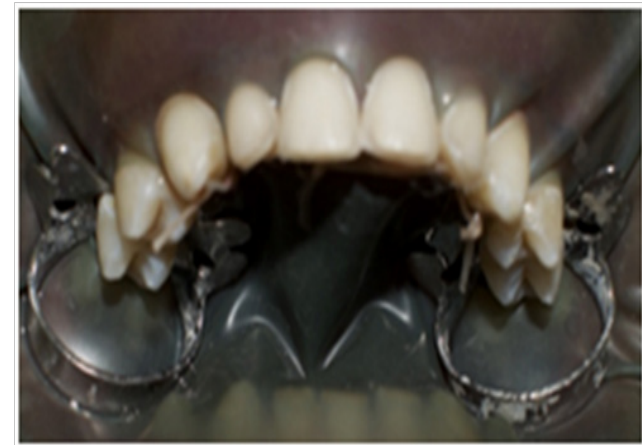

Figure 6 Rubber dam application.

a. Conditioning of the ceramic veneers:

The internal surfaces were etched using $9 \%$ hydro fluoride acid (HF) for 60 s, then rinsed and thoroughly cleansed. Placing the veneers into a container of $95 \%$ alcohol and putting it into an ultrasonic bath for four minutes was crucial for the removal of any impurities that may interfere with the adhesion mechanism.

Thus, the internal veneer surfaces looked clear and had similar appearance of etched enamel. Then, the silane coupling agent was applied followed by the five-second evaporation of its solvents and activated by hot air. Just before placing the veneers, a bonding agent was applied without photo polymerization (Figure 7).

b. Conditioning the prepared teeth:

A 37\% phosphoric acid etching gel was applied for 30seconds, as the preparation was within enamel (Figure 8), then rinsed off with water and dried until getting a frosty white appearance (Figure 9). Adhesive agent was applied and photopolymerized (Figure 10).

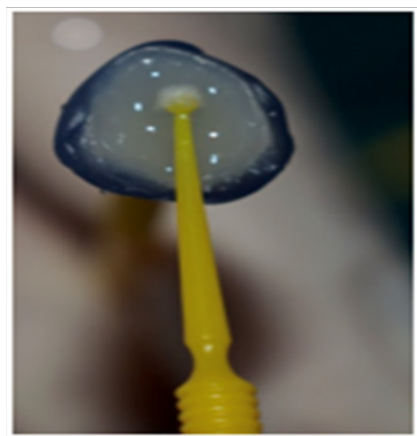

Figure 7 adhesive agent application with w micro-brush.

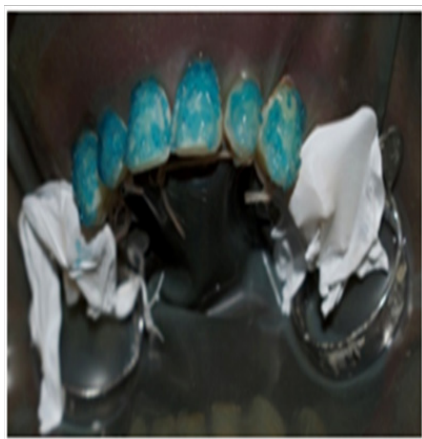

Figure 8 37\% phosphoric acid application during 30seconds.

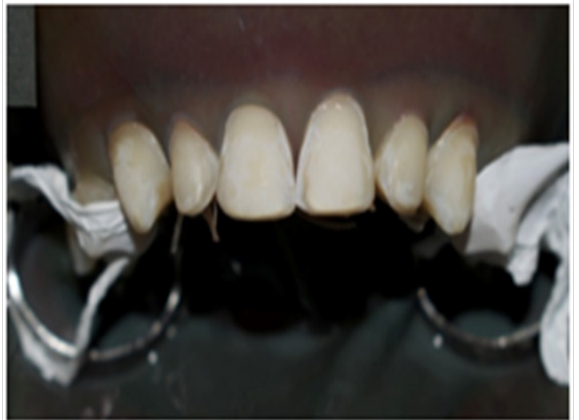

Figure 9 Frosty white appearance confirming well etched enamel.

Light curing composite resin was used for cementation. Veneers were seated starting from central incisors where a maximum of symmetry was required. Every veneer was gently positioned with finger pressure. Correct seating was ascertained by checking the marginal fits. Initial light cure lasted five seconds. Then excess of luting agent was removed. A final photopolymerization was performed for 60seconds on the buccal and lingual surface of each restoration (Figure 11). Residual cement elimination was thoroughly assessed by probing the crevicular sulcus. Dental floss passes with light friction. 
Static and dynamic occlusion was scrutinized. Minor adjustments were realized by means of fine diamond bur. Finally, the margins of the bonded veneers were finished and polished. The patient was satisfied with both esthetic and functional outcome (Figure 11) (Figure 12).

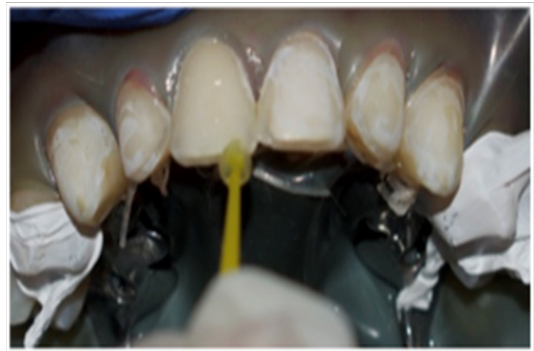

Figure 10 Adhesive agent application with a micro-brush

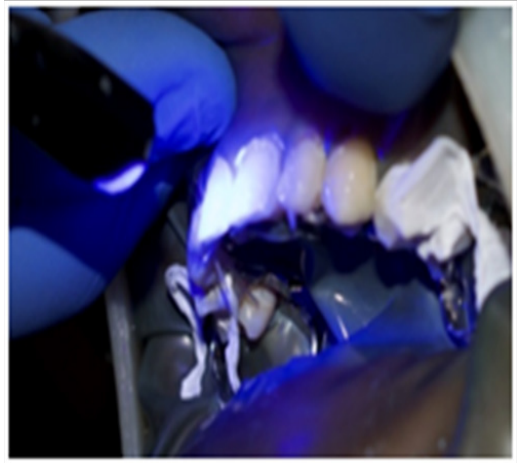

Figure I I Photopolymerization.
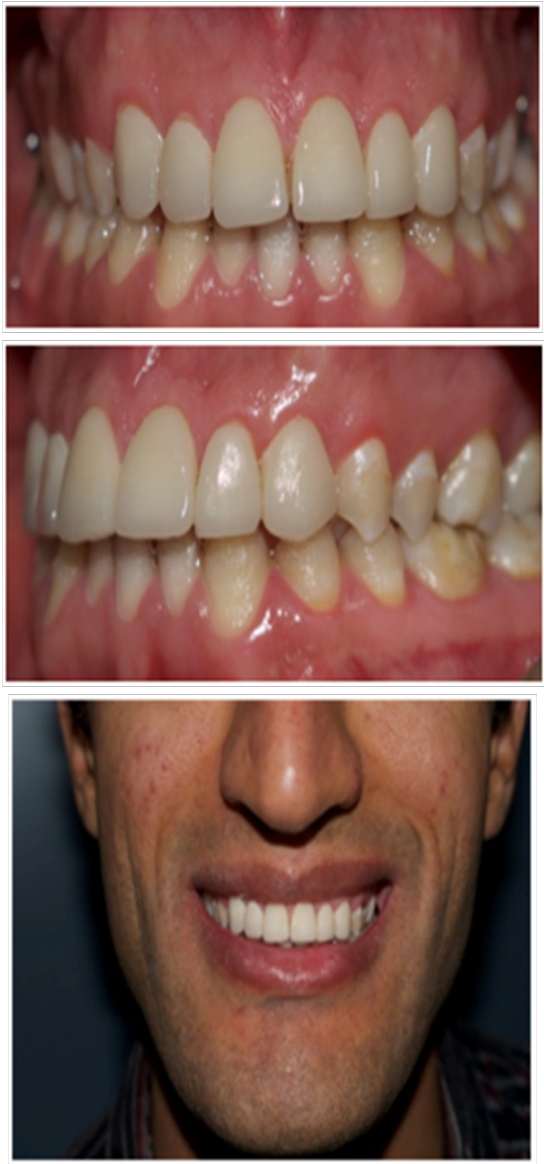

Figure 12 Final result: a: intra oral view, b: lateral view, c smile.

\section{Recall checks}

Regular recalls were programmed during which oral hygiene maintenance and gingival margins stability were evaluated.

\section{Discussion}

The article illustrates a minimally invasive approach to optimize the smile after an orthodontic treatment by managing teeth size discrepancies. Several studies ${ }^{5-10}$ have shown that the estimated survival of ceramic veneers over a period of 10years exceeds $90 \%$. Thus, porcelain veneers are deemed conservative and predictable. To guarantee longevity and satisfactory outcome, their indication must be precise and the clinical protocol must be detailed.

Prior to any esthetic restorative treatment, periodontal health is crucial. Apart from the oral hygiene enhancement, a mucogongival plastic surgery proved necessary to reestablish shallow sulci, enlarge crown heights, correct gingival margins and zeniths positions. Thus, improving the gummy smile appearance. A gingivectomy procedure without osseous resection was indicated as the sulci were not deep on probing. Aiming to obtain biomimetic restorations with excellent biomechanical and optical properties, ceramics are undoubtedly the material of choice. ${ }^{9}$

When considering the fracture resistance, ceramic systems have evolved throughout the years. It was evident that the fracture resistance of $0.5 \mathrm{~mm}$ thick veneers increases following an efficient adhesive cementation. ${ }^{11}$ Lithium dislocate are selected thanks to their less porosity and higher crystalline content compared to other ceramics. ${ }^{12,13}$ The flexural strength of lithium disilicate veneer is approximately $360 \mathrm{MPa}^{8}$ which is sufficient to rehabilitate the anterior guidance. ${ }^{13,14}$

Even with a relatively high crystalline content, causing a relatively low refractive index, this material is still indicated for anterior restorations. ${ }^{12}$ Currently, numerous translucency blocks (HT: hight translucency, LT: low translucency, MO: medium opacity) with two shapes and several colors (20 colors for HT and LT and 5 for MO) are manufactured. ${ }^{14}$ Lithium disilicate veneers are processed through CAD/CAM systems allowing thus highly-precise predictable results. ${ }^{14,15}$

After getting the patient approval, the restorative procedure is started. Before tooth preparation, shade must carefully be selected regarding underlying tooth color, luting cement and the opacity translucency of the ceramic used which are key parameters to be considered as they all contribute to the final color. ${ }^{2}$

Early protocols were in favor of "no tooth preparation" technique. However, Pini et al., ${ }^{16}$ demonstrated a reduced retention capacity of the aprismatic enamel surface. Moreover, Heymann et al., ${ }^{17}$ proved that intra enamel preparation removes the outer layer of enamel which is rich in fluoride that may be more resistant to acid etching and creates a rough surface for improved bonding.

Therefore, current studies support conservative preparation restricted to enamel as it provides a better seat and more effectively diminishes marginal leakage. Galip et al. ${ }^{18}$ showed a $99 \%$ veneer survival rate with enamel preparation versus $94 \%$ for veneers with enamel only at the margins.

Tooth preparation should be meticulously performed because of its impact on the longevity of the restorations reducing, thus, the risk of failure ${ }^{10}$ Enough space for the restorative material must be managed to prevent crack onset inside the veneers. ${ }^{9}$ Although several 
techniques were proposed to assess the substance reduction during teeth preparation, the use of depth cuts/grooves deemed more precise to avoid excessive preparation. ${ }^{2}$ Incisal edge is beveled from buccal to palatal providing better seating, stronger veneers and allowing easy characterization by the ceramist. ${ }^{2,19,20}$ During the bonding protocol, the use of rubber dam isolation is recommended. So that a contamination of enamel bonding surface is avoided, a correct visualization of the gingival margins and a clear restorative environment are allowed and an excess of cement is easily removed..$^{14}$ The precementation chemical treatment by acid etching and silanization increases the wettability of resin cement. This enhances retention, reduces internal cracks propagation and increases fracture resistance of the veneers after adhesive cementation..$^{21,22}$

Light curing composite resin is the cement of choice. As it has a longer working time than dual cure or chemically cured composite, it offers sufficient time to remove excesses before curing. This facilitates the finishing procedures. ${ }^{2}$ Moreover, light curing composite has no amines. This is critical to final color restoration stability. ${ }^{14}$ Due care must be paid to the follow up recalls to determine the longevity of the restorations.

\section{Conclusion}

Optimization and predictability of the disilicate veneers restorations must not be taken for granted. Comprehensive treatment planning, meticulous material choice and extreme precision in scheduling every step of the treatment are cardinal rules to success with laminate veneers. Further long-term clinical trials are necessary to study their marginal integrity, marginal staining and their effect on surrounding tissues and evaluate the mechanical performance of lithium disilicate for the fabrication of veneers. A perfect coordination between the dental team and the patient, taking into account the latter's wishes and expectations, is crucial.

\section{Acknowledgements}

None.

\section{Conflict of interest}

The author declares no conflict of interest.

\section{References}

1. Giselle Rodrigues R, Ana Laura Rezende V, Fernanda Pereira S. Minimally invasive approach in esthetic dentistry: composite resin versus ceramics veneers. Bioscience Journal. 2017;33(1):1-9.

2. Mancini M, Maurizio M. Ceramic veneers: a step-by-step case report. Global Journal of Oral Science. 2016;2(2016):20-27.

3. Imburgia M, Canale A, Cortellini D, et al. Minimally invasive vertical preparation design for ceramic veneers. Int J Esthet Dent 2016;11(4):460-471.

4. Rafael de almeida decurcio, Paula de carvalho cardoso. Porcelain laminate veneers: A minimally invasive esthetic procedure. Stomatos. 2013;17(33):1-8.

5. Peumans M, De Munck J, Fieuws S, et al. A prospective ten-year clinica trial of porcelain veneers. Journal of Adhesive Dentistry. 2004;6(1):6576.
6. Beier US, Kapferer I, Burtscher D, et al. Clinical performance of porcelain laminate veneers for up to 20years. Int $J$ of Prosthodont. 2012;25(1):79-85

7. Tajammul A. Porcelain laminate veneers:a minimally invasive esthetic procedure. Journal of Evolution of Medical and Dental Sciences. 2013;2(45):8856-8860.

8. Beier US, Kapferer I, Burtscher D, et al. Clinical performance of porcelain laminate veneers for up to 20years. Int $J$ of Prosthodont. 2012;25(1):79-85.

9. Veneziani M. Ceramic laminate veneers:clinical procedures with a multidisciplinary approach. The international journal of esthetic dentistry. 2017;12(4):426-448

10. Elif O, Şükran B. Survival of porcelain laminate veneers with different degrees of dentin exposure: 2-year clinical results. Journal of Adhesive Dentistry. 2014;16(5):1-9.

11. Marina Studart A, Diana Ferreira Gadelha D, Rafael Massunari M. Reestablishment of esthetics with minimum thickness veneers:a one-year follow-up case report. Quintessence Internationa. 2014;45(7):593-597.

12. DA Cunha, Pedroche LO, Gonzaga CC, et al. Esthetic, occlusal, and periodontal rehabilitation of anterior teeth with minimum thickness porcelain laminate veneers. J prosthetic Dent. 2014;112(6):1315-1318.

13. Fons-Font A, Solá-Ruíz MF, Granell-Ruíz M, et al. Choice of ceramic for use in treatments with porcelain laminate veneers. Med Oral Patol Oral Cir Bucal. 2006;11(3):297-302.

14. Durán Ojeda, Gerardo, Henríquez Gutiérrez, et al. A step-by-step conservative approach for CAD-CAM Laminate Veneers. Case reports in dentistry. 2017.

15. Beuer F, Schweiger J, Edelhoff D. Digital dentistry:an overview of recent developments for CAD/CAM generated restorations," British Dental Journal. 2008;204(9):505-511.

16. Pini NP, Aguiar FH, Lima DA, et al. Advances in dental veneers: materials, applications, and techniques. Clinical, Cosmetic and Investigational Dentistry. 2012;4:9-16.

17. Heymann HO, Swift EJ, Ritter AV. Sturdevant's Art and Science of Operative Dentistry. 6th ed. St.Louis: USA: Elsevier Mosby; 2013. p. 624-688.

18. Gurel G, Sesma N, Calamita MA, et al. Influence of enamel preservation on failure rates of porcelain laminate veneers. Int J periodontics Restorative Dentistry. 2013;33(1):31-39.

19. Da Costa DC, Coutinho M, de Sousa AS, et al. A meta-analysis of the most indicated preparation design for porcelain laminate veneers. $J A d-$ hes Dent. 2013;15(3):215-220.

20. Mital P, Kathryn D, Paul AB. Porcelain laminate veneers. What, when and how? Dental Nursing. 2010;6(10).

21. Burke FJ. Survival rates for porcelain laminate veneers with special reference to the effect of preparation in dentin:a literature review. $J$ Esthet Restor Dent. 2012;24(4):257-265.

22. Dileep S, Agarwal P, Rakesh J, et al. All ceramic veneers: a case report. Journal of evolution of medical and dental sciences-jemds. 2016;5(54):3705-3708. 